\title{
Design and Research of VR System in Latin Dance Teaching
}

\author{
Yanghui Xu, Yi Li \\ Chongqing Real Estate College, Chongqing, China
}

Keywords: Latin dance skills, VR simulation system, movement trajectory

\begin{abstract}
Today, the integration of VR technology with teaching process is becoming a new research direction. In this paper, information technology is used in Latin dance classes to capture the skilled movements of teachers and students. Through teaching video data storage and movement trajectory comparison, the skills and techniques of Latin dance can be showed more directly and insightfully, which is benefit for monitoring the teaching course, controlling exercise intensity more reasonably, improving students' visionary and hearing perception of Latin dance, and enhancing the teaching effects. At the same time, the application of virtual environment and virtual dance partners in the teaching procedures can arouse students' learning interests, and promote the scientific development of Latin dance.
\end{abstract}

\section{Introduction}

Integrating techniques about computer graphics, multimedia, three-dimensional display, human-computer interaction, sensors, network and artificial intelligence, Virtual Reality technology provides users with a three-dimensional space generated by the computer simulation system; in this virtual world, users can experience simulated feelings of vision, force sensing, hearing, touching and movement. [1] Through creating a realistic simulation environment, users can immerse in the virtual world and interact with the simulated environment. In recent years, the combination of VR technique and teaching process is becoming a new research direction. The University of Nevada established a 640 square foot VR + AR studio, named @ Reality, in the Mathewson - IGT Knowledge Center. School officials expressed, @ Reality studio would provide the university community with special space, equipments, software and professional knowledge which can help students to explore the technology of VR and AR properly [2]. The purpose of this paper is to explore the application of VR technology in the teaching process of Latin dance. By using VR simulation technology, the movement trajectory of marker points in teachers' and students' bodies are captured to establish virtual human models. Through comparison after data entry, skills of Latin dance can be represented more intuitively.

The combination of VR technology and Latin dance teaching can make the teaching process more scientific and interesting, and enhance students' understanding of Latin dance skills, as well as their total level of Latin dance. Thank you for Yan $\mathrm{Xu}$, the founder and general manager of T-Sense VR laboratory for introducing the principles of VR simulation system in detail, and providing important technical guidance for this research.

\section{Overview of Latin Dance.}

\subsection{Introduction to Latin dance}

After the World War I, dancers in Europe studied the court dance and Latin America dance; after scientific, standard and systematic combination, standard dance steps were issued in 1925, and evolved into various contemporary dance sports in the end. [3] Dance sports can be divided into two groups: modern dance and Latin dance. Modern dances include waltz, tango, brisk, fox-trot and Viennese waltz. Latin dance, also known as the social dance, include the graceful rumba dance, the vivacious cha-cha dance, the amusing cowboy dance, the passionate samba dance and the forceful 
paso doble dance. Latin dance has distinctive characteristics of passion, romantic and energetic; it is a lively form of art expression.

\subsection{Characteristics of Latin dance}

Dancers need more endurance and strength since there are a lot of rotations and diversified technical movements in Latin.

The music rhythms of rumba and paso doble are moderate; while samba, cha-cha and cowboy dance are fast paced.

\section{Design of VR Simulation System in Latin Dance Classes}

\subsection{Overall structure design}

In combination with VR technology, the author plans to design the Latin dance simulation system from three aspects: movement trajectory generation system, whole-process data monitoring and storage system and virtual dance partner design system.

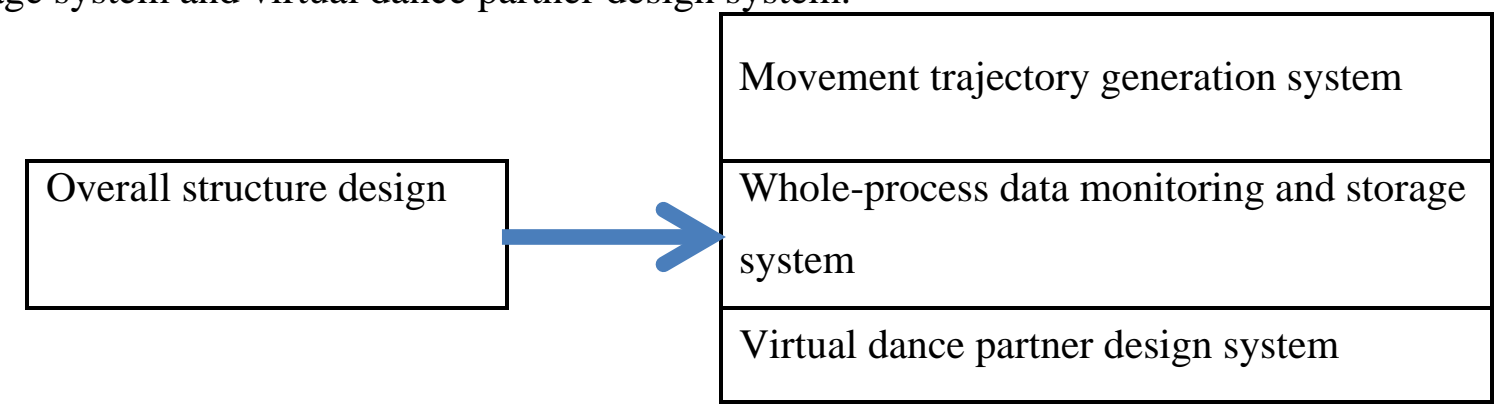

\subsection{Overview of the three systems}

Movement trajectory generation system. On the basis of computer vision principles, optical motion capture technology can be used to monitor and track the movement of feature points of the target through multiple high-speed cameras located in different positions (as shown in Figure 1). According to different types of sensors, optical motion capture can be divided into unmarked optical motion capture and marked optical motion capture. [4] When learning Latin dance, the relationship between weight, center of gravity and movement must be determined. The weight means body weight, namely the pressure of body on the floor; the center of gravity refers to the center of the vertical line of three-dimensional human body pressure. [5] Latin dance has higher requirements on dancers' qualities, including speed, power, endurance, flexibility, balance and other aspects. There are also requirements on the tension of thigh and foot pressure. These dance skills cannot be totally grasped merely from the external forms of body. Mere visual capture may bring about wrong information. It is also the difficult point in the traditional teaching process of Latin dance: body feelings are difficult to be spotted by naked eyes, or clearly expressed through language. It is hard to judge whether the postures and muscle tensions are correct. In the process of Latin teaching, the marked optical motion capture technology can be used. Marker points can be pasted in the key parts of human body, such as joints, hip, waist and abdomen, legs, feet and ankles (shown in Figure 2). In the movement process, such as box step and cha-cha forward check step, optical cameras can record the tracks of reflective markers (marker points) of human body from different angles. Maker points help to measure, track and record the movement of abdomen, back, thighs, hip and feet. Timely and accurately recorded data are input into the computer software for processing. In the virtual three-dimensional space, human bodies are reconstructed to present the moving postures of various body parts in box and check; an "adjustable digital model of posture" is constructed through joints and connecting rods of sensors with angel. [6] Simulation of human body can make the teaching process more intuitive. Then, the same method is used to capture students' movement and compare relevant data with the correct movement trajectory. The movements of students can be checked in this way. Meanwhile, students can learn the right movement skills more intuitively, and correct the wrong movements consciously; thus, they can form the correct dynamic stereotype. Their learning abilities, as well as the controlling 
of Latin dance skills, can be improved quickly and efficiently.

In the process, teachers and students need to be kept in consistent datum marks and orientation to obtain accurate data.

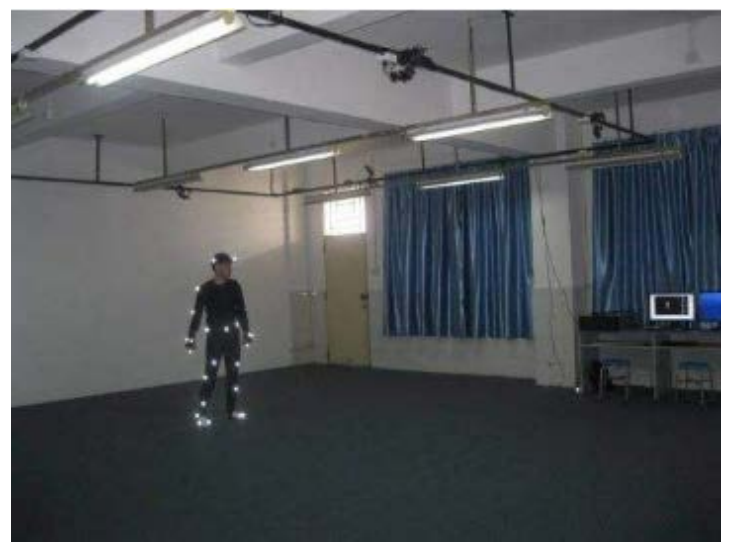

Figure 1. High-speed cameras

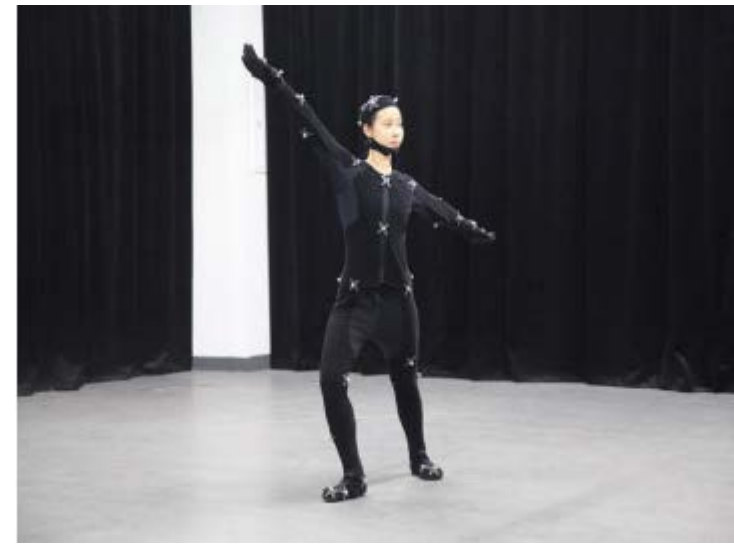

Figure 2. Marker points

This article takes the teaching of backward steps in rumba as an example. In this step, the left leg serves as power leg, while the right foot serves as motion leg. Rumba music has four-count rhythm. In the second beat, students need to withdraw their right foot to the back. But they tend to open the right hip back at the same time. In the third beat, they need to push left hip forward. Another proper movement is to move the gravity center to the back foot (right foot) in the second beat, and then to the left foot at the third beat. In the second beat, their hips need to move forward straightly without turning. The groin move forward, with center of gravity falls between two feet. In the third beat, they need to use the pressure of left foot on the floor to push the right hip forward, up and turn back. Marker points are labeled in hips, connection parts of hip and thigh, inner thighs, heels, feet, toes and other places. At that time the bodies are in movement; students can see the power parts of muscles.

Another example is forward check step in cha-cha. In the learning process, students are easily to be misled by visual disturbance. In the second beat, students tend to relax hips; their bodies can be sinking. Dancers with scattered forces are unable to show the features of cha-cha dance: agile and fast movement. They need to use right back thigh (Marker point) to thrust forward and push hip (Marker point) forward. They cannot relax inguinal (Marker point) and pelvic area (Marker point); these parts need to move slightly forward. At the same time their hips (Marker point) and inner thighs (Marker point) need to be engaging. The energy of the whole body should be concentrated within their bodies like spirals. The extension of other actions, such as shoulder extrusion and arm swing, is generated naturally through force generation.

In above examples, if the movement skills can be tracked through VR optical motion capture technology, and the movement trajectory can be captured by Marker points, reaction forms between hips and the middle of body [5], spine and hips, as well as the back thigh and knee in Latin dance will be clearly shown. Students can form more in-depth understandings about core skills of Latin dance.

Whole-process data monitoring and storage system. Entry of data about teaching process and storage analysis.

Data about captured movement trajectory are recorded, compared and analyzed by computers. Relevant data are stored to provide reference for the improvement of teaching process.

Monitoring the teaching process and making the evaluation system more scientific. VR technology in combination with the Internet is capable of collecting information in the teaching process, recording students' physical conditions in detail, scientific monitoring students training amount, duration and content, so as to fully master their physiological loads in training, provide feedbacks according to related data, explore improvement methods in Latin teaching, enhance students' physical beauty, and gradually reduce teaching loads to ensure the scientific and rational teaching content.

At the same time, along with the improvement of social recognition of Latin dance, it is necessary to further improve dancers' artistic accomplishments. VR technology can be used to capture the 
expressions and eye contacts of professional Latin dancers in games and performances, and obtain their body and facial movements. Thus, the expressive forces of top Latin dancers can be showed more clearly, which will stimulate students' learning interests and improve their art accomplishments.

Designing of virtual partners and enhancement of partner cooperation effects. Using VR technology, students can carry on training and performances in the virtual environment of dancing floor, which is helpful in improving their Latin dance skills and stimulating their artistic expressions. The fusion of emotion in Latin dance can arouse students' learning interests to the greatest extent. In the Latin dance for two people, the male dancer plays an important role of "guiding". For the female dancer, guiding signs are essential suggestions in direction indication and action cohesion. Guided by man, the two dancers can form closed position, lateral lines position, lateral position, relative lateral position, fraction position, fractional side position, fan position, shadow position, inhibited lateral position and many other kinds of body positions, which can present the space beautifulness of Latin dance. But in the teaching process, due to age characteristics of students, they are ashamed to express themselves in front of partners of the opposite sex; they are usually too nervous, which will lead to stiff movements, even embarrassments or conflicts in cooperation. Through designing virtual partners, beginners can avoid embarrassments and conflicts. When they have mastered necessary skills, real partners can be arranged to improve the collaboration effects and show more perfect dances, like romantic rumba and humorous cha-cha. Yan $\mathrm{Xu}$, the general manager of $\mathrm{T}$ - Sense VR laboratory puts forward, it is technically feasible to capture the whole body movement and record human postures, and then produce virtual partners. But the problem of real human feelings is still waiting to be solved. Even in fields where VR technology is relatively mature, such as vehicle maintenance and repair, unreal body feelings are inevitable in virtual interactions.

\section{Conclusions and Suggestions}

VR technology can effectively monitor the teaching process of Latin dance. Through capturing movement trajectory and collecting information, students' training status, including the amount of training, training duration and training content can be scientifically and effectively monitored. Timely feedbacks of teaching effect are conductive to improving teaching qualities. Now optical motion capture technology is relatively mature; interference of cables and equipment connecting lines can be eliminated. Teachers and students can present their postures freely. Marker points are cheap enough to be widely promoted. The VR technology simulated dancing floor and constructed virtual figures can be used to expand students' horizons, so as to enable them to immerse in the virtual reality environment [7], and stimulate enormous passion for learning.

To sum up, VR technology develops rapidly, and still has broad application space. It is of great significance to integrate VR technology with Latin dance teaching process.

\section{References}

[1] L.W. Gao, Analysis of three-dimensional animation and virtual reality technology, J. Technology and Arts. 12 (2017).

[2] L.K. Xiao, University of Nevada sets up VR+AR studio to create campus VR/AR [EB/OL]. (2017-10-11). [2017-12-04]. http://www.heix.cn/news/a6105.html.

[3] J.J. Cai, Research on the phenomenon of "plateau" in the special training of sports dance and its prevention, Shandong Normal University, 2014.

[4] H.Y. Huang, L.L. Tu, Development of optical motion capture technology in film and television animation, J. Policy Research \& Exploration. 8 (2016).

[5] S.H. Xi, Graphic analysis on technical theory and skills of rumba, People's Sports Press, Beijing, 2011.

[6] M.L. Zhou, Study on human posture reconstruction based on mark points, College of Computer Science and Electronic Engineering, 2013.

[7] X.H. Li, J.J. Pei, Application of VR technology in physical education, J. Research on computer application teaching. 6 (2011). 